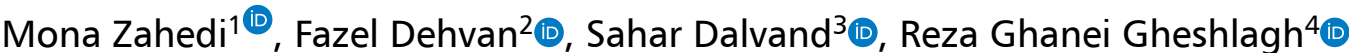 \\ ${ }^{1}$ Student Research Committee, Kurdistan University of Medical Sciences, Sanandaj, Iran \\ ${ }^{2}$ Department of Nursing, Faculty of Nursing and Midwifery, Kurdistan University of Medical Sciences, Sanandaj, Iran \\ ${ }^{3}$ Functional Neurosurgery Research Center, Shohada Tajrish Comprehensive Neurosurgical Center of Excellence, Shahid Beheshti University \\ of Medical Sciences, Tehran, Iran \\ ${ }^{4}$ Spiritual Health Research Center, Research Institute for Health Development, Kurdistan University of Medical Sciences, Sanandaj, Iran
}

\title{
Examination of the relationship of
} knowledge of diabetes, attitude toward diabetes, and health literacy with diabetes management self-efficacy using hierarchical
multiple regression modeling

\section{ABSTRACT}

Background. Self-efficacy in diabetes management can empower patients and result in their more active participation in the treatment process. In addition, a patient's behavior is influenced by their knowledge of and attitude toward their illness. Therefore, the goal of the present study was to examine the association of knowledge of diabetes, attitude toward diabetes, and health literacy with diabetes management self-efficacy in patients with type 2 diabetes.

Methods. This descriptive-correlational study was conducted in 2019. The sample included 115 patients with diabetes ( 59 women and 56 men) attending diabetes centers of Sanandaj, Kurdistan, Iran, who were selected using a convenience sampling method. The Brief Diabetes Knowledge Test (DKT), the Diabetes Attitude Scale (DAS), the Health Literacy for Iranian Adults (HELIA) scale, and the Diabetes Management Self-Efficacy Scale (DMSES) were used to gather data. The data was

Address for correspondence:

Reza Ghanei Gheshlagh

Kurdistan University

of Medical Sciences

Pasdaran Ave, Sanandaj 6618634683, Iran

Phone: +98 9144050284

Fax: +98 36237511

e-mail: Rezaghanei30@gmail.com

Clinical Diabetology 2020, 9; 6: 394-399

DOI: $10.5603 /$ DK.2020.0048

Received: 23.06.2020

Accepted: 02.11.2020 analyzed using descriptive statistics, Pearson correlation coefficient, and stepwise regression analysis. All the analyses were perfumed using SPSS, version 16 . The significance level was set at $\mathbf{0 . 0 5}$ for all the tests. Results. The mean age of participants was $46.61 \pm$ 13.73 years. Health literacy $(r=0.585)$ and attitude $(r=0.396)$ were significantly correlated with self-efficacy $(P=0.001)$. According to the results of stepwise regression analysis, $34.3 \%$ and $36.6 \%$ of the variance of self-efficacy was explained by health literacy alone and health literacy together with attitude, respectively. One standard deviation change in health literacy and attitude was associated with 0.51 and 0.71 standard deviation change in self-efficacy, respectively. Conclusion. Health literacy and attitude toward diabetes are positively associated with self-efficacy in diabetes management, and improving these variables can improve self-efficacy in patients with type 2 diabetes. (Clin Diabetol 2020; 9; 6: 394-399)

Key word: diabetes, self-efficacy, knowledge, attitude, health literacy

\section{Introduction}

Diabetes is the most common chronic metabolic disease that has become a silent epidemic in today's world [1]. In 2014, about 387 million people around the world had diabetes, and it has been projected that by 2035,529 million people will have this disease 
[2]. According to statistics, diabetes control is not at the desired level globally, and a significant increase is projected to occur in the global prevalence of diabetes. This is especially concerning for Middle-East countries; it has been projected that in near future, Iran will have the highest prevalence of diabetes in Middle-East after Pakistan [3]. The prevalence of diabetes in Iran is $9.3 \%$ [4]; however, given the fact that a lot of diabetic patients are not aware of their condition, the prevalence of diabetes seems to be higher than the reported prevalence rates [5]. Currently, more than 3 million people in Iran have diabetes, and if effective measures are not taken to address this problem, this number will be doubled by 2030 [6].

Diabetes management is complex and requires measures beyond blood sugar control, and a broad range of interventions are needed to improve the outcomes for this group of patients [7]. Improvement of self-care skills in diabetes like any other chronic disorder requires behavioral changes in patients [2]. Self-efficacy is an important factor in diabetes management, and can lead to increased quality of life, reduced risk of hospitalization, and less risky behaviors in diabetic patients [8]. Self-efficacy includes self-motivated behaviors allowing a patient to understand factors influencing their condition, make effective decisions, and engage in effective behaviors to improve their own health [6]. Patients with higher levels of self-efficacy tend to actively participate in self-care programs, and are more successful in managing their symptoms [8]. Self-efficacy empowers patients and increases their sense of control; therefore, diabetic patients with higher levels of self-efficacy feel more control on their problem, and try harder to adhere to treatment [9]. Huang et al. [10] found that self-efficacy was the strongest predictor of adherence to treatment in diabetic patients. Diabetic patients have the most important role in managing their illness; therefore, their level of knowledge of diabetes is an important factor in the effective management of their symptoms [11].

Knowledge is the best protective factor against diabetes, so that Moodley [12] considers it to be the most effective weapon against diabetes. Patients with adequate knowledge of diabetes are more likely to take responsibility for their own health [13], and have a greater sense of empowerment [14]. Attitude motivates behavior, and any change in the attitude of patients results in changes in their behaviors [15]. In addition to knowledge and attitude, health literacy may also be related to diabetes management self-efficacy. Health literacy is defined in terms of cognitive or social skills determining one's motivation or ability to obtain, understand, or use information in order to maintain or improve their own health [16]. Herath maintains that health literacy is an integral part of diabetes management [13]. Higher levels of health literacy allow diabetic patients to better understand information related to proper diets, insulin injection, blood sugar control, and acceptance of the disease, and increase the participation of patients in making decisions about treatment methods [17]. Schilinger [18] found that patients with adequate health literacy had more control over their blood sugar levels and experienced fewer diabetes complications compared to those with inadequate levels of health literacy.

Knowledge, attitude, and health literacy are influenced by culture; therefore, the present study is aimed at examining the relationship of knowledge of diabetes, attitude toward diabetes, and health literacy with diabetes management self-efficacy in patients with type 2 diabetes attending diabetes centers of Sanandaj, Kurdistan, Iran in 2019.

\section{Methods}

This was a descriptive-correlational study. The statistical population included all patients with type 2 diabetes attending diabetes centers of Sanandaj in 2019, and the sample included 115 patients who were selected for this population, using a convenience sampling method. Sample size was calculated based on the following formula:

$$
N=\left(\frac{Z_{1-\alpha / 2}+Z_{1-\beta}}{0.5 \ln \frac{1+r}{1-r}}\right)^{2}+3,
$$

according to $\alpha=0.05$ and a correlation coefficient of $r=0.3$.

The inclusion criteria were willingness to participate in the study, medical record in the diabetes center, aged 16-65 years, and ability to read and write. Incomplete questionnaires were discarded. The data was collected using the demographic questionnaire, the Brief Diabetes Knowledge Test (DKT), the Diabetes Attitude Scale (DAS), the Health Literacy for Iranian Adults (HELIA) scale, and the Diabetes Management Self-Efficacy Scale (DMSES).

The Brief Diabetes Knowledge Test (DKT): This test was developed by Fitzgerald et al. [19] at Michigan Diabetes Research Center. It has 23 items with four response options, one of which is the correct answer (scored 1) and the rest are incorrect answers (scored 0 ). Total score ranges from 0 to 23, with higher scores indicating higher diabetes knowledge $[19,20]$. Because a Persian version of the test was not available, after obtaining permission from the original developers of the test, it was translated into Persian using the forward-backward method, and 10 experts in nursing confirmed its qualitative content validity. Then, the dif- 
ficulty and discrimination coefficients were examined. 10 items with difficulty coefficients below 0.2 were discarded, and the reliability of the final scale with 16 items was found to be 0.661 , using the KuderRichardson coefficient.

The Diabetes Attitude Scale (DAS): This scale was translated into Persian and validated in Iran by Mahjouri et al. [21]. It has 33 items that are rated on a 5-point Likert-type scale ranging from 1 (totally disagree) to 5 (totally agree), and higher scores indicate better attitude toward diabetes.

The Health Literacy for Iranian Adults (HELIA) scale: This scale was developed by Montazeri et al. [22], and has 33 items that are rated on a 5-point Likert-type scale.

The Diabetes Management Self-Efficacy Scale (DMSES): This scale was translated into Persian and validated in Iran by Haghayegh et al. [23]. It has 19 items rated on a 10-point Likert-type scale ranging from 1 (I cannot at all) to 10 (I surely can). Higher scores on this scale indicate greater self-efficacy in diabetes management. The reliability of DAS, HELIA, and DMSES questionnaires was $0.752,0.896$, and 0.853 , respectively.

After explaining the objectives of the study to the participants and obtaining their consents for participation, the questionnaires were administered. It should be noted that the participants were not required to write their real names on the questionnaires. The present study was approved by the ethics committee at Kurdistan University of Medical Sciences (no. IR.MUK. REC.1398.212)

\section{Statistical analysis}

The data was described using percentage, mean, and standard deviation. Independent t-test and oneway analysis of variance were used to compare quantitative data between two groups and more than two groups, respectively. Chi-squared test and Pearson correlation coefficient were used to examine the relationship between qualitative data and the relationship between quantitative data, respectively. Stepwise multiple regression was used to determine the important factors in predicting diabetes management self-efficacy. Significance level was set at 0.05 for all the tests. All the analyses were performed using SPSS, version 16 .

\section{Results}

The study sample included 115 patients with type 2 diabetes (59 women and 56 men) with a mean age of $46.61 \pm 13.73$ years. The mean disease duration was $9.24 \pm 5.67$ years. Further details are provided in Table 1.
Table 1. Demographic properties of the participants

\begin{tabular}{lll}
\hline Variable & N & $\%$ \\
\hline Gender & 56 & 48.7 \\
Male & 59 & 51.3 \\
$\quad$ Female & & \\
Marital situation & 18 & 15.7 \\
$\quad$ Single & 97 & 84.3 \\
$\quad$ Married & & \\
Literacy & 70 & 60.9 \\
$\quad$ Primary & 29 & 25.2 \\
$\quad$ High school & 16 & 13.9 \\
$\quad$ University & & \\
Employment status & & \\
Employed & 67 & 58.3 \\
Un employed & 48 & 41.7 \\
Drug & & \\
Insulin & 47 & 40.9 \\
Pills & 12 & 48.7 \\
Insulin + pills & 56 & 10.4 \\
\hline
\end{tabular}

According to the results of Pearson correlation coefficient, self-efficacy in diabetes management was significantly associated with attitude $(r=0.396)$ and health literacy $(r=0.585)$. In other words, better attitude toward diabetes and higher health literacy were related to higher self-efficacy in diabetes management $(P<0.001)$ (Table 2).

Stepwise regression analysis was employed to determine the predictive power of knowledge of diabetes, attitude toward diabetes, and health literacy in diabetes management self-efficacy. According to Pearson correlation results, health literacy and attitude were significantly related to self-efficacy; therefore, the two variables were included in the regression model to predict self-efficacy. Health literacy was included in the first step, and health literacy and attitude were included in the second step. In the first step, health literacy significantly predicted self-efficacy $(F=58.883$, $P=0.001)$, and explained $34.3 \%$ of the variance of this variable. In the second step, health literacy and attitude together explained $36.6 \%$ of the variance of self-efficacy ( $F=32.366, P=0.001)$. In this step, attitude alone explained $2.3 \%$ of the variance of selfefficacy (Table 3).

As shown in the table above, regression coefficients indicate that one standard deviation change in health literacy and attitude is associated with 0.510 and 0.172 standard deviation change in self-efficacy, respectively. Regression coefficients also show that health literacy $(P=0.001)$ and attitude $(P=0.001)$ have a significant positive effect on self-efficacy. In other words, higher 
Table 2. Correlation coefficients between diabetes management self-efficacy with knowledge of diabetes, attitude toward diabetes, and health literacy in patients with type 2 diabetes

\begin{tabular}{lcccccc}
\hline Variables & $\mathbf{M}$ & SD & Knowledge & Attitude & Health literacy & Self-efficacy \\
\hline Knowledge & 7.41 & 2.82 & - & & & \\
Attitude & 121.07 & 9.28 & 0.18 & - & & \\
Health literacy & 134.69 & 12.70 & 0.05 & $0.44^{*}$ & - & - \\
Self-efficacy & 152.56 & 24.93 & 0.02 & $0.396^{*}$ & $0.585^{*}$ & - \\
\hline
\end{tabular}

$* \mathrm{P}<0.001$

Table 3. The results of stepwise multiple regression

\begin{tabular}{llcccccccc}
\hline Model & Source of changes & SS & $\mathbf{d f}$ & $\mathbf{M S}$ & $\mathbf{F}$ & $\mathbf{R}$ & $\mathbf{R}^{2}$ & $\mathbf{A}_{\mathrm{dj}} \mathbf{R}^{2}$ & $\mathbf{P}$ \\
\hline Health literacy & Regression & 24277.783 & 1 & 24288.783 & 58.88 & 0.585 & 0.343 & 0.337 & 0.001 \\
& Residual & 46590.478 & 113 & 412.305 & & & & & \\
& Sum & 70868.261 & 114 & & & & & & \\
Health literacy & Regression & 25957.064 & 2 & 12978.532 & 32.36 & 0.605 & 0.366 & 0.355 & 0.001 \\
\& Attitude & Residual & 44911.197 & 112 & 400.993 & & & & & \\
& Sum & 70868.261 & 114 & & & & & \\
\hline
\end{tabular}

Table 4. Stepwise regression analysis for variables predicting diabetes management self-efficacy

\begin{tabular}{ccccccc}
\hline Model & Variable & \multicolumn{2}{c}{ Unstandardized coefficients } & Standardized coefficients & \multirow{2}{*}{ t } & \multirow{2}{*}{$\mathbf{P}$} \\
\cline { 3 - 5 } & & B & Standard error & Beta & & \\
\hline 1 & (constant) & -2.095 & 20.244 & & -0.104 & 0.918 \\
& Health literacy & 1.148 & 0.150 & 0.585 & 7.674 & 0.001 \\
2 & (constant) & -37.882 & 26.540 & & -1.427 & 0.156 \\
& Health literacy & 1 & 0.164 & 0.510 & 6.079 & 0.001 \\
& Attitude & 0.461 & 0.255 & 0.172 & 2.046 & 0.001 \\
\hline
\end{tabular}

health literacy and a better attitude toward diabetes are associated with higher diabetes management selfefficacy (Table 4).

\section{Discussion}

The goal of the present study was to examine the association of knowledge of diabetes, attitude toward diabetes, and health literacy with diabetes management self-efficacy. According to the study results, diabetes management self-efficacy was significantly associated with attitude and health literacy. In addition, health literacy and attitude explained $34.3 \%$ and $2.3 \%$ (together $36.6 \%$ ) of the variance of diabetes management self-efficacy, respectively. Osborn et al. [24] also found a significant positive association between self-efficacy and health literacy in diabetic patients. Masoompour et al. [25] also showed a significant positive relationship between health literacy and self-efficacy in patients with diabetes. Sedighi Pashaki et al. [26] maintain that proper management of diabetes requires multi- disciplinary cooperation among healthcare providers from different fields and also cooperation between healthcare providers and patients, and that the concept of health literacy is highlighted with a greater focus on patient-centered care and patient empowerment. Diabetic patients with enough health literacy are less likely to have problems in reading medication labels and health-related educational brochures, interpreting medical test results, and providing informed consent for medical procedures [27]. They are also more able to manage their illness effectively. On the other hand, the relationship between health literacy and self-efficacy is not limited to diabetes, and is also observed in other chronic disorders. For example, a study among patients with colorectal cancer showed a significant relationship between health literacy and patient self-efficacy [28]. Bohanny et al. [29] found that receiving diabetes education, job status, and health literacy explained $11.8 \%$ of the variance of self-efficacy in patients. 
We found no significant association between knowledge and self-efficacy. In contrast with this finding, Abedini [11] maintains that patients' lack of knowledge of their own conditions can increase the prevalence of diabetes complications. Santos also considers knowledge to be one of the most important factors in diabetes management, because the patient needs to have enough knowledge about diabetes treatments, role of carbohydrates, and proper diets [30]. Patients with enough knowledge about their illness are more likely to take responsibility for their own health [13]. In the present study, the diabetic patients obtained less than half of the total knowledge score that seems inadequate. Murata believes that lack of knowledge of diabetes undermines the ability of diabetic patients to manage their illness [14]. Therefore, we cannot expect from patients with insufficient knowledge of their illness to show self-efficacy in diabetes management; this indicates the importance of providing patients with proper training. Given the fact that patients' knowledge of their conditions is influenced by cultural factors, learning readiness, cognitive performance, family support, and barriers to care [23], proper training programs should be provided for diabetic patients, so that they can be empowered to effectively manage their illness. Economic problems in Iran can affect patients' attitudes toward diabetes. Even if patients have good knowledge about the disease, when they cannot afford adequate treatment of the disease, this knowledge cannot help them. It seems that knowledge can be effective when the patient has sufficient financial support. On the other hand, seeing other diabetics with lower limb amputation, blindness, kidney failure, and dialysis dependence makes these patients who do not yet have the complications of diabetes pay more attention to self-care behaviors to better manage their disease.

In the present study, attitude toward diabetes was a moderate predictor of self-efficacy in diabetes management. Consistent with this finding, previous studies have shown a significant relationship between attitude and diabetes management self-efficacy [31, 32]. Given that attitude can be influenced by knowledge, this finding can be attributed to the participants' low knowledge of diabetes. Patients with inadequate knowledge of factors related to their illness, including its causes and types, proper diets, and proper exercise, cannot have the right attitude toward their conditions [33]. Attitude motivates behavior, and having a proper attitude can change patients' behaviors and improve their self-efficacy [15]. It seems that the emotional nature of the Kurds was clearer in this study because attitude (not knowledge) had an effect on the selfefficacy of their disease management.
One important limitation of the present study was that the data was gathered using self-report questioners. Therefore, participants' answers to the questionnaires may have been influenced by their mental and emotional state. Iran is a country of different cultures and ethnicities, and this study was conducted on Kurds living in western Iran. The results of this study can be generalized to all Kurdish populations in Iran, but since these concepts are influenced by the culture and context of different societies, it is recommended to study in Arab, Turkish, Lor, Balouch, Lak and Fars cultures to be able to comment on this with more confidence.

\section{Conclusion}

Overall, the study results indicated the positive association of diabetes management self-efficacy with health literacy and attitude toward diabetes in patients with type 2 diabetes. Therefore, self-efficacy of diabetic patients in managing their own illness can be improved through designing strategies to improve their health literacy and attitude toward diabetes.

\section{Acknowledgments}

This study was part of an approved research proposal in Kurdistan University of Medical Sciences. In this regard, the researchers thanked the Research Deputy of Kurdistan University of Medical Sciences for approving this proposal.

\section{Conflict of interest}

The authors declare no conflict of interest.

\section{REFERENCES}

1. Pashaki MS, Mezel JA, Mokhtari Z, et al. The prevalence of comorbid depression in patients with diabetes: A meta-analysis of observational studies. Diabetes Metab Syndr. 2019; 13(6): 3113-3119, doi: 10.1016/j.dsx.2019.11.003, indexed in Pubmed: 31790965.

2. Tharek Z, Ramli AS, Whitford DL, et al. Relationship between self-efficacy, self-care behaviour and glycaemic control among patients with type 2 diabetes mellitus in the Malaysian primary care setting. BMC Fam Pract. 2018; 19(1): 39, doi: 10.1186/ s12875-018-0725-6, indexed in Pubmed: 29523075.

3. Olfatifar M, Karami M, Shokri P, et al. Prevalence of chronic complications and related risk factors of diabetes in patients referred to the Diabetes Center of Hamedan Province. Scientific Journal of Hamedan Nursing and Midwifery Faculty. 2017; 25(2): 69-74, doi: 10.21859/nmj-25029.

4. Haghdoost AA, Rezazadeh-Kermani M, Sadghirad B, et al. Prevalence of type 2 diabetes in the Islamic Republic of Iran: systematic review and meta-analysis. East Mediterr Health J. 2009; 15(3): 591-599, indexed in Pubmed: 19731775.

5. Shomali M, Mohamadi S, Ervanloo S. An investigation of nurses' knowledge regarding diabetes Zanjan University of Medical Sciences. Journal of Diabetes Nursing. 2015; 3(1): 59-68.

6. Rahimi MIN. Rezvan Madani F, Eghbalian A. Knowledge and practice level of self-directed care among diabetics in Kermanshah 
City in 2014: A Short Report. The Journal of Rafsanjan University of Medical Sciences. 2015; 14(2): 167-72.

7. Shahraki RA, Kamrani AA, Sahaf R, et al. Effects of Nationwide Program for Prevention and Control of Diabetes initiated by the ministry of health on elderly diabetic patients' knowledge, attitude and practice in Isfahan. Salmand. 2019: 84-95, doi: 10.32598/sija.14.1.84

8. Hatef M, Sharif Nia H, Mousavinasab N, et al. Self-efficacy and prediction of associated factors in patients with chronic diseases. Journal of Mazandaran University of Medical Sciences. 2018; 28(162): 86-94.

9. Niguse H, Belay G, Fisseha G, et al. Self-care related knowledge, attitude, practice and associated factors among patients with diabetes in Ayder Comprehensive Specialized Hospital, North Ethiopia. BMC Res Notes. 2019; 12(1): 34, doi: 10.1186/s13104019-4072-z, indexed in Pubmed: 30658687.

10. Huang YM, Shiyanbola OO, Smith PD. Association of health literacy and medication self-efficacy with medication adherence and diabetes control. Patient Prefer Adherence. 2018; 12: 793-802, doi: 10.2147/PPA.S153312, indexed in Pubmed: 29785094.

11. Abedini Z. Shouri Bidgoli A, Ahmari Tehran H. Study of knowledge and practice of patient self directed care among diabetics patients. Qom Univ Med Sci J. 2008; 2(2): 37-42.

12. Moodley LM, Rambiritch V. An assessment of the level of knowledge about diabetes mellitus among diabetic patients in a primary healthcare setting. South African Family Practice. 2014; 49(10), doi: 10.1080/20786204.2007.10873652.

13. Herath HMM, Weerasinghe NP, Dias $\mathrm{H}$, et al. Knowledge, attitude and practice related to diabetes mellitus among the general public in Galle district in Southern Sri Lanka: a pilot study. BMC Public Health. 2017; 17(1): 535, doi: 10.1186/s12889-017-4459-5, indexed in Pubmed: 28571566.

14. Murata GH, Shah JH, Adam KD, et al. Factors affecting diabetes knowledge in Type 2 diabetic veterans. Diabetologia. 2003; 46(8): 1170-1178, doi: 10.1007/s00125-003-1161-1, indexed in Pubmed: 12856126.

15. Sheeran $P$, Maki A, Montanaro $E$, et al. The impact of changing attitudes, norms, and self-efficacy on health-related intentions and behavior: A meta-analysis. Health Psychol. 2016; 35(11): 1178-1188, doi: 10.1037/hea0000387, indexed in Pubmed: 27280365.

16. Zahedi S, Darvishpoor Kakhaki A, Hosseini M, et al. The correlation between self-care and health literacy in patients undergoing hemodialysis. Iranian Journal of Diabetes and Lipid Disorders. 2018; 17(4): 180-188.

17. Abbaszadeh Bazzi M, Karimiaval M. Relationship between health literacy and self-care behaviors in diabetic patients type ii referred to the center of diabetes control and prevention in Zabol. Journal of Health Literacy. 2018; 3(1): 10-19.

18. Schillinger D, Grumbach K, Piette J, et al. Association of health literacy with diabetes outcomes. JAMA. 2002; 288(4): 475-482, doi: 10.1001/jama.288.4.475, indexed in Pubmed: 12132978.

19. Fitzgerald JT, Funnell MM, Anderson RM, et al. The reliability and validity of a brief diabetes knowledge test. Diabetes Care. 1998; 21(5): 706-710, doi: 10.2337/diacare.21.5.706, indexed in Pubmed: 9589228.
20. University of Michigan Health System: Michigan Diabetes Research Center (MDRC); 2019. http://diabetesresearch.med.umich.edu/ Tools_Surveylnstruments.php.

21. Mahjouri MY, Esfahani E, Larijani B. Evaluation of psychometric properties of the third version of the Iranian Diabetes Attitude Scale (IR-DAS-3). Iranian Journal of Diabetes and Lipid Disorders. 2011; 10(1): 1-6.

22. Montazeri A, Tavousi $M$, et al. Health Literacy for Iranian Adults (HELIA): development and psychometric Properties. Payesh. 2014; 13(5): 589-599.

23. Haghayegh AS, Ghasemi N, Neshatdoost HT, et al. Psychometric Properties Of Diabetes Management Self-Efficacy Scale (Dmses). IJEM. 2010; 12(2): 111-115.

24. Osborn CY, Cavanaugh K, Wallston KA, et al. Self-efficacy links health literacy and numeracy to glycemic control. J Health Commun. 2010; 15 Suppl 2: 146-158, doi: 10.1080/10810730.2010.499980, indexed in Pubmed: 20845200.

25. Masoompour M, Tirgari B, Ghazanfari Z. The Relationship between health literacy, self-efficacy, and self-care behaviors in diabetic patients. Evidence Based Care. 2017; 7(3): 17-25.

26. Pashaki MS, Eghbali T, Niksima SH, et al. Health literacy among Iranian patients with type 2 diabetes: A systematic review and meta-analysis. Diabetes Metab Syndr. 2019; 13(2): 1341-1345, doi: 10.1016/j.dsx.2019.02.020, indexed in Pubmed: 31336489.

27. Saeed H, Saleem Z, Naeem R, et al. Impact of health literacy on diabetes outcomes: a cross-sectional study from Lahore, Pakistan. Public Health. 2018; 156: 8-14, doi: 10.1016/j.puhe.2017.12.005, indexed in Pubmed: 29353668.

28. von Wagner C, Semmler C, Good A, et al. Health literacy and self-efficacy for participating in colorectal cancer screening: The role of information processing. Patient Educ Couns. 2009; 75(3): 352-357, doi: 10.1016/j.pec.2009.03.015, indexed in Pubmed: 19386461.

29. Bohanny W, Wu SFV, Liu CY, et al. Health literacy, self-efficacy, and self-care behaviors in patients with type 2 diabetes mellitus. J Am Assoc Nurse Pract. 2013; 25(9): 495-502, doi: 10.1111/17457599.12017, indexed in Pubmed: 24170654.

30. Santos FR, Bernardo V, Gabbay MAL, et al. The impact of knowledge about diabetes, resilience and depression on glycemic control: a cross-sectional study among adolescents and young adults with type 1 diabetes. Diabetol Metab Syndr. 2013; 5(1): 55, doi: 10.1186/1758-5996-5-55, indexed in Pubmed: 24289093.

31. Goodarzi M, Ebrahimzadeh I, Rabbi A, et al. Asghari jafarAbadi M. The relationship between knowledge, attitude and practice with self-efficacy in type 2 diabetic patients in Karaj. Iranian Journal of Diabetes and Lipid Disorders. 2012; 11(3): 269-81.

32. Reisi M, Mostafavi F, Javadzade SH, et al. Assessment of some predicting factors of self-efficacy in patients with type 2 diabetes. Iranian Journal of Endocrinology and Metabolism. 2015; 17(1): 44-52.

33. Abolghasemi $R$, Sedaghat $M$. The patient's attitude toward type 2 diabetes mellitus, a qualitative study. J Relig Health. 2015; 54(4): 1191-1205, doi: 10.1007/s10943-014-9848-9, indexed in Pubmed: 24599712. 